\title{
Efficacy of Anti-Fungal Agents Incorporated in Tissue Conditioners in Inhibiting the Growth of Candida albicans
}

\author{
Vidya S. Bhat ${ }^{1}$, Varsha P. Bhat ${ }^{2}$, Anurag Agarwal ${ }^{3}$, Sanath Shetty ${ }^{4}$ \\ 1, 2, 3, 4 Department of Prosthodontics, Yenepoya Dental College, Mangaluru, Karnataka, India.
}

\section{ABSTRACT}

\section{BACKGROUND}

Chronic atrophic candidiasis is an inflammation of the oral cavity. Because of the diverse possible origins of denture stomatitis, several treatment procedures are used, like antifungal therapy, correction of ill-fitting dentures and effective plaque control. Tissue conditioners (porous in nature, tend to harbor bacteria) have been used to enhance adaptation of the removable denture prosthesis and aid in recovery of denture bearing tissues. A lot of complete denture wearers, owing to their age, lack the manual dexterity to maintain adequate denture hygiene. Incorporating antifungal agents in tissue conditioners may be a useful method of drug delivery to overcome the above-mentioned obstacles for effective treatment. This in vitro study tests the efficacy of 2 antifungal drugs, fluconazole and ketoconazole incorporated in 2 tissue conditioners (Viscogel and GC Soft) in inhibiting the growth of Candida albicans.

\section{METHODS}

This is an experimental study of two antifungal agents (fluconazole and ketoconazole) in powdered form which were incorporated into powder of the tissue conditioner in a specific concentration and tested against a culture of Candida albicans. The mixed tissue conditioner was injected into $6 \mathrm{~mm}$ diameter holes, punched in the plates of the culture medium. Then the plates were incubated and the inhibition diameter for each sample was measured after $24 \mathrm{hrs}$. Readings were subjected to statistical analysis.

\section{RESULTS}

Controls did not show any zone of inhibition. GC Soft with ketoconazole combination had a mean of $27.97 \mathrm{~mm}$; GC Soft with fluconazole showed a mean of 25.97. The combination of Viscogel with ketoconazole showed a mean diameter of inhibition of $26.07 \mathrm{~mm}$; the combination of Viscogel and fluconazole showed a mean of $27.33 \mathrm{~mm}$ and was non-significant.

\section{CONCLUSIONS}

There was no inhibition of Candida albicans observed in Viscogel and GC Soft controls. The antifungal agents ketoconazole and fluconazole combined with Viscogel and GC soft showed definite zone of inhibition indicating positive inhibitory effect on Candida albicans.

\section{KEY WORDS}

Anti-Fungal, Tissue Conditioner, Chronic Atrophic Candidiasis
Corresponding Author: Dr. Vidya S. Bhat, Yenepoya Dental College, Affiliated to Yenepoya (Deemed to Be) University, University Road, Deralakatte, Mangaluru - 575018, Karnataka, India.

E-mail:vidya.bhat@yenepoya.edu.in

DOI: $10.14260 / j e m d s / 2020 / 856$

How to Cite This Article:

Bhat VS, Bhat VP, Agarwal A, et al. Efficacy of anti-fungal agents incorporated in tissue conditioners in inhibiting the growth of candida albicans. J Evolution Med Dent Sci 2020;9(52):3904-3908, 10.14260/jemds/2020/856

Submission 03-07-2020,

Peer Review 04-11-2020,

Acceptance 11-11-2020,

Published 28-12-2020.

Copyright (C) 2020 JEMDS. This is an open access article distributed under Creative Commons Attribution License [Attribution 4.0 International (CC BY 4.0)] 


\section{BACKGROUND}

Removable prosthesis placed in the oral cavity produces noticeable changes in the oral environment that may have an unwanted effect on the soundness of oral tissues. The direct sequelae include mucosal reactions, residual ridge resorption etc.

One of the major mucosal reactions is Candida associated denture stomatitis. The inflammation is associated with Candida albicans.

Chronic atrophic candidiasis is an inflammation of the oral cavity that prevails in a large number of denture patients. Its prevalence is more common in the institutionalized denture wearing population, up to $88 \% .{ }^{1}$ In this condition, chronic irritation of the mucosal tissues in contact with the denture base results to observable discrete areas of pinpoint inflammation often associated with the openings of the palatal mucous glands, or as inflammation of an area covered by the maxillary denture.

Accumulation of Candida in the area between dentures and the oral mucosa causes this type of denture stomatitis. The condition is not a specific disease entity because other factors such as bacteria, mechanical irritation and allergy may be seen during an event of stomatitis. Due to the varying possible origins of denture stomatitis, several treatment procedures like correction of ill-fitting dentures, antifungal therapy and effective plaque control are used. Candida albicans infected patients should be treated with antimycotic medications. Advising the patient to discontinue the denture is the first step in the treatment of denture stomatitis. But this is not very practical from functional and social view point, so alternatives are necessary.

Tissue conditioners have been utilised to enhance adaptation of the denture and to allow recovery of denture bearing area by providing a temporary or permanent cushion like effect. These materials are generally classified into two types - short term soft liners (tissue conditioners) and longterm soft liners.

Antifungal agents can also be prescribed to hasten the recovery. Although topical agents are quite effective, they are often associated with poor patient compliance due to frequency of doses, lack of comprehension and objectionable taste (e.g. Nystatin). If unresponsive to topical agents, only then systemic medications are adopted as a mode of therapy due to their increased risk of serious side effects. Topical nystatin, amphotericin B and miconazole were the drugs of choice for oral candidiasis therapy. Systemic triazole antifungal drugs, fluconazole and itraconazole were developed later which became an important addition in the treatment of this infection. ${ }^{2}$

These therapies are difficult to implement in the hospital setting as several patients have sensory loss, compromised comprehension and manual adeptness and depend on caretakers to follow the drug administration protocol. Combining antifungal agents into tissue conditioners may be a propitious method of drug delivery to overcome this hurdle for therapy.

The main advantages of using antifungal agents in tissue conditioners as a mode of drug delivery are i) economical- a smaller quantity of the antifungal agent is utilized compared to regular therapy, ii) patient compliance- not needed, iii) concomitant treatment of Candidal infection as well as abused denture bearing area, iv) decreased frequency of application.

Douglas and Walker in 1973 suggested incorporating antifungal agents into tissue conditioners as a treatment modality. They investigated the combined effect of nystatin with tissue conditioners and observed their effect as fungicide in differing degrees. Another study on ketoconazole, miconazole and nystatin added to different tissue conditioners showed $100 \%$ efficacy in inhibiting Candida albicans. ${ }^{3}$

There is a need to find different methods for local drug delivery. This in vitro study tests the efficacy of antifungal drugs, fluconazole and ketoconazole incorporated into 2 commercially available tissue conditioners (Viscogel and GC Soft) in inhibiting the growth of Candida albicans.

\section{METHODS}

\section{Tissue Conditioners}

These are usually dispensed as a powder liquid system. The powder contains a polymer, a polymethylmethacrylate (PMA), or its copolymers, and the liquid contains a mixture of ethyl alcohol (solvent) and an aromatic ester (dibutyl phthalate), which acts as a plasticizer that lowers the glass transition temperature (Tg) of the polymer rendering it a soft gel. Both Viscogel and GC Soft are commercial acrylic based tissue conditioner and the drugs used are ketoconazole and fluconazole.

\section{Test Isolates}

Fungi selected for testing - American Type Culture Collection (ATCC) 10231 of Candida albibans, Microbiologics.

\section{Inoculum Preparation}

Candida albicans ATCC was obtained from HiMedia, Mumbai. The lyophilized culture was inoculated into Brain Heart Infusion (BHI) broth and incubated overnight at $37^{\circ} \mathrm{C}$. Subculture was done from the BHI broth culture onto Sabouraud's Dextrose Agar (SDA) and the plates were incubated at $37^{\circ} \mathrm{C}$, for 24 hours.

Suspensions of Candida culture obtained on SDA plates were made up in saline to get turbidity equivalent to 0.5 McFarland standards (which is equal to 106 - 108 organisms / $\mathrm{mL}$. Further sterile saline was added to the suspension, so as to obtain 103 - 104 organisms / mL.

\section{Preparation of Culture Plates}

100 microliters of the inoculum was dropped on each sterile Sabouraud agar plate and a lawn culture was made. The plates were incubated at $37^{\circ} \mathrm{C}$ for 72 hours at humidity of 75 $\%$. These conditions simulate the oral environment in which the organisms flourish.

\section{Preparation of Samples}

$6 \mathrm{~mm}$ diameter holes (cores) were punched in the plates of the culture medium. Antifungal agents fluconazole and 
ketoconazole in tablet forms were crushed into powder and tissue conditioner was hand mixed in a specific concentration of $5 \% \mathrm{wt} / \mathrm{wt}$ ( $0.95 \mathrm{~g}$ base $+0.05 \mathrm{~g}$ antifungal agent).

Then the mixture was combined with the liquid of the tissue conditioner as per the manufacturer's instructions. The mixed tissue conditioner was injected into the cores using a disposable syringe and plates were incubated. Inhibition diameter for each sample was measured at $24 \mathrm{hrs}$. using a standardized geometrical divider. Maximum inhibition diameter was calculated by subtracting the core diameter from the raw data. Data was subjected to statistical ANOVA analysis.

\section{RESULTS}

The controls did not show any zone of inhibition. GC Soft with ketoconazole combination had a mean of $27.97 \mathrm{~mm}$; GC Soft with fluconazole showed a mean of 25.97. The combination of Viscogel with ketoconazole showed a mean diameter of inhibition of $26.07 \mathrm{~mm}$; the combination of Viscogel and fluconazole showed a mean of $27.33 \mathrm{~mm}$ and were nonsignificant.

\section{Control Groups}

Pure culture

- Viscogel 10. (Samples)

- G C Soft 10.

\section{Test Combinations}

- Ketoconazole with GC Soft 30.

- Ketoconazole with Viscogel 30.

- $\quad$ Fluconazole with GC Soft 30.

- $\quad$ Fluconazole with Viscogel 30.

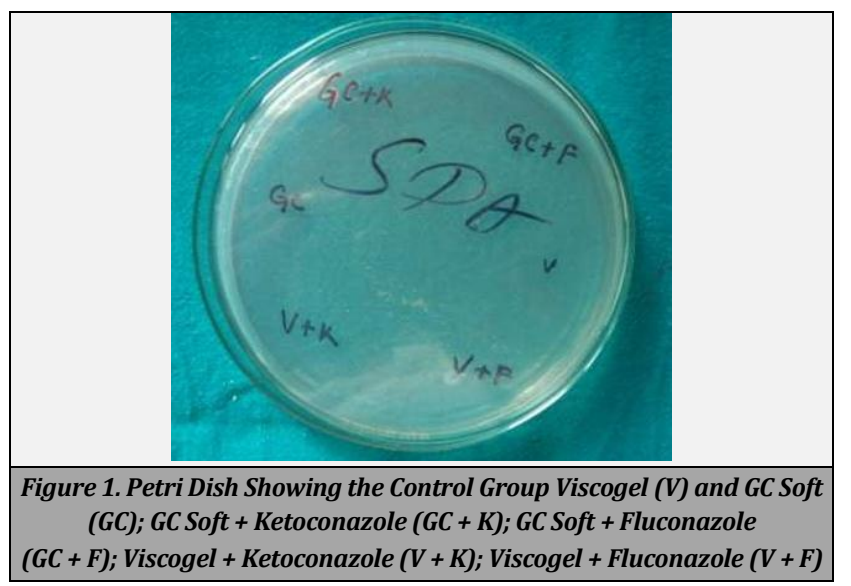

There is no inhibition of Candida albicans observed in Viscogel and GC Soft controls. The controls GC Soft and Viscogel alone did not show any zone of inhibition. GC Soft with ketoconazole combination (30) had a mean of $27.97 \mathrm{~mm}$ whereas GC Soft with fluconazole showed a mean of $25.97 \mathrm{~mm}$. The $\mathrm{p}$ value $<0.0001$ shows a highly significant relation between the study and the control groups. (Table 1)

Similarly, the combination of Viscogel with ketoconazole showed a mean diameter of inhibition of $26.07 \mathrm{~mm}$, whereas the combination of Viscogel and fluconazole showed a mean of
$27.33 \mathrm{~mm}$. The $\mathrm{p}$ value determined is $<0.0001$ thus it is highly significant.

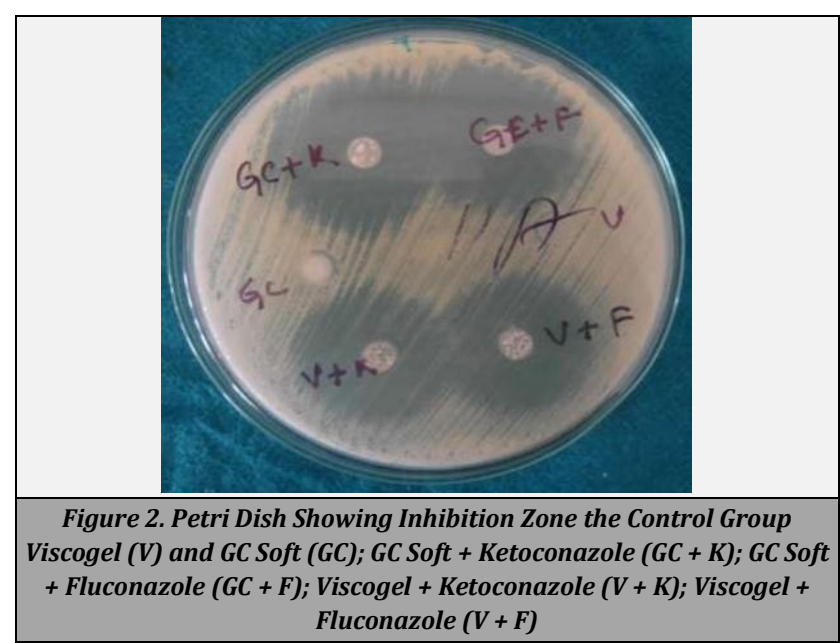

\begin{tabular}{|cccccc|}
\hline Drug & & N & Min. & Max. & Mean \\
GC & Control & 10 & 0 & 0 & 0.00 \\
(diameter & Ketoconazole & 30 & 18 & 34 & 27.97 \\
in mm) & Fluconazole & 30 & 20 & 34 & 25.97 \\
& Total & $\mathbf{7 0}$ & $\mathbf{0}$ & $\mathbf{3 4}$ & $\mathbf{2 3 . 1 1}$ \\
Viscogel Control (Diameter in mm) & 10 & 0 & 0 & 0.00 \\
& Ketoconazole & 30 & 11 & 36 & 26.07 \\
& Fluconazole & 30 & 19 & 32 & 27.33 \\
& Total & $\mathbf{7 0}$ & $\mathbf{0}$ & $\mathbf{3 6}$ & $\mathbf{2 2 . 8 9}$ \\
\hline & Table 1. Comparison of Ketoconazole and \\
& Fluconazole Only with the Controls \\
\end{tabular}

The combination of GC + ketoconazole shows mean inhibition diameter of 28.50, GC + fluconazole 25.97, Viscogel + ketoconazole 26.07 and Viscogel + fluconazole 27.33 (Table 2) which were non-significant ( $p$ value $>0.001$ ). The combination of GC + ketoconazole showed a slight wider range of diameter of inhibition in relation to GC + fluconazole and Viscogel + fluconazole showed a slight wider diameter of inhibition in relation to Viscogel + ketoconazole combination. (Table 2).

\begin{tabular}{|cccccc|}
\hline Drug and Material & $\mathbf{N}$ & Min. & Max. & Mean & S.D. \\
GC + Ketoconazole & 30 & 18 & 34 & 27.97 & 3.409 \\
GC + Fluconazole & 30 & 20 & 34 & 25.97 & 4.089 \\
Viscogel + Ketoconazole & 30 & 11 & 36 & 26.07 & 5.860 \\
Viscogel + Fluconazole & 30 & 19 & 32 & 27.33 & 3.594 \\
Total & $\mathbf{1 2 0}$ & $\mathbf{1 1}$ & $\mathbf{3 6}$ & $\mathbf{2 6 . 8 3}$ & $\mathbf{4 . 3 7 6}$ \\
\hline Table 2. Comparison among the Different Combinations of Tissue \\
Conditioners (Viscogel and GC Soft) and the Antifungal Agents \\
Used (Ketoconazole and Fluconazole) (Diameter in mm) \\
\hline
\end{tabular}

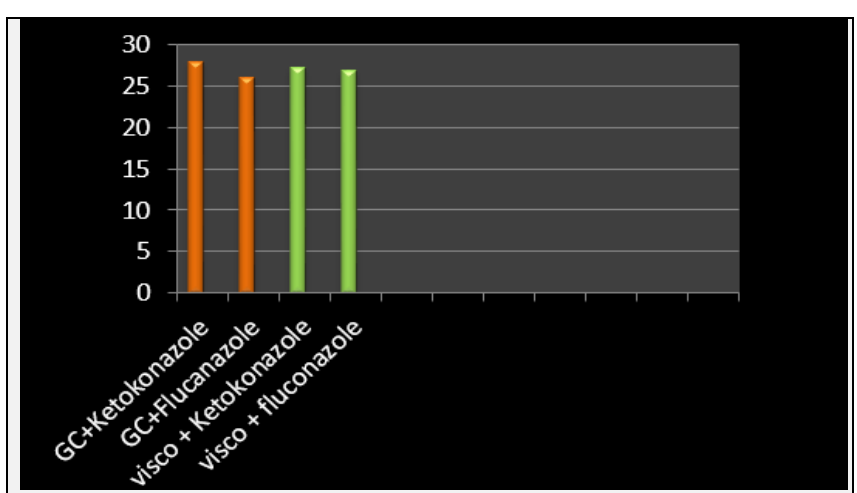

Figure 3. Comparison of the Four Different Combinations in Relation to Each Other 


\section{DISCUSSION}

Chronic atrophic candidosis or denture stomatitis is a common type of fungal infection, seen in more than half of denture wearing population and usually found on the palatal mucosa beneath the intaglio surface of the maxillary denture. Although it affects both complete and partial denture wearers, it is rarely seen on mucosa underneath the lower denture. Usually denture stomatitis is present without any symptoms, but common signs and symptoms presenting maybe bleeding of mucosa, halitosis, swelling, dryness in the mouth and unpleasant taste. ${ }^{2}$ The associated symptoms like burning or other painful sensations from the oral mucosa are seen, in patients whom the infection has spread to other areas of the oral cavity or the pharynx and in those with an increased risk of systemic fungal infections due to other debilitating diseases, medications or radiation therapy. ${ }^{3}$

Effective treatment must target the multifarious aetiology of the disease. The therapy should involve the oral hygiene instructions, elimination of anatomical irregularities, substitution of old prostheses, nutritional restoration, establishment of a non-traumatic occlusion, antifungal treatment and systemic evaluation.

Treatment with antifungal therapy should be utilized in patients after the clinical diagnosis has been confirmed by a mycological analysis.

Combining antifungal agents into tissue conditioners may be a propitious method of drug delivery to overcome this hurdle for therapy. The main advantages of using antifungal agents in tissue conditioners as a mode of drug delivery are - i) Economical- a smaller quantity of the antifungal agent is utilized compared to regular therapy, ii) Patient Compliancenot needed, iii) Concomitant treatment of Candidal infection as well as abused denture bearing area, iv) Decreased frequency of application. ${ }^{4}$

In a previous in vitro study, where $1 \mu \mathrm{g} / \mathrm{mL}$ of miconazole in $33 \%$ collagen solution was used on resin discs, the growth of $C$. albicans on the discs was nearly completely inhibited. ${ }^{5}$

Antifungal agents used in the form of suspensions or crushed tablets have been studied earlier.6,7 Studies have also used antifungal agent susceptibility discs dipped in tissue conditioners to check the efficacy. Although a study which showed hand mixing itraconazole into Viscogel dissolved it completely within 3 minutes, authors quoted that this combination is not recommended for clinical use. ${ }^{7}$

As antifungal drugs have to be incorporated by the manufacturer into the tissue conditioners, this method of crushing the drug and incorporating it into the tissue conditioners used in the present study Viscogel and GC Soft. The two antifungal agents used in the present study are: (a) ketoconazole (b) fluconazole.

The ATCC (American Type Control Collections) strain of Candida albicans was used in the present study. Candida albicans is the most common and widely obtained organisms from the smear of a denture stomatitis patient, up to $59.2 \%{ }^{8}$ Although Candidal commensals are shown to be predominant in denture stomatitis according various studies, there exists divergent Candida species and biotypes, constituting $1 / 4^{\text {th }}$ of all the yeast isolates, which should not be disregarded. However, Candida albicans being the most common to isolate was used in the present study.
The present study utilizes the drug dosage of 5 micrograms and same concentration or even higher concentration of the inoculum of Candida albicans used in previous studies.6,7,9

In our study, Viscogel and GC Soft were not successful in inhibiting Candida albicans, when used alone. The results obtained were expected and in accordance with an earlier study wherein the authors stated the complete inertness of Viscogel and was not recommended for use without antifungal agents in the treatment of denture stomatitis along with a fungal infection. ${ }^{6}$

Both the tissue conditioners i.e. Viscogel and GC Soft do not have any antifungal property of their own.

In our study, both the ketoconazole and fluconazole combinations with both of the tissue conditioners i.e. Viscogel and GC Soft were found to be effective against Candida strain.

Ketoconazole combined with either with GC Soft or Viscogel had similar zone of inhibition and was nonsignificant $(p>0.001)$. Same result was seen with fluconazole with ( $p<0.001)$ indicating that the combination of fluconazole when mixed with Viscogel or GC Soft yielded a zone of inhibition which did not show extremes of values. Also, all the combinations used in the study were nonsignificant among each other.

The inhibition of antifungal drug effect by mixing preweighed amount of drug into tissue conditioners is significant $(p<.001)$ in all the combinations used. This might be a result of interaction between the antifungal drug and the tissue conditioner. The present study is in contrast with those studies which used suspensions. ${ }^{4,6}$

The maximum zone of inhibition was recorded at 24 hours after which the effect of the antifungal drugs gradually decreased in the following days, with the culture media being contaminated with other bacterial organisms. Thus, limiting the readings only up to the third day. The reading in the present study conforms to the readings acquired in earlier studies. ${ }^{6}$

In the clinical scenarios, if usage of Viscogel alone in the treatment of denture stomatitis will imply a purely physical voiding of trauma. Depending upon the presence or absence of fungal infection and the unresponsiveness of the fungi to traditional treatment, anti-fungal therapy inclusion is decided. In positive cases, combination of 500,000 $\mathrm{U}$ nystatin into the Viscogel is indicated. Though the tissue conditioner loses its visco-elastic properties within few days, this amount of nystatin's effectiveness remains. ${ }^{6}$

This extended effectiveness of nystatin may be exhausted earlier than expected as clinical scenario varies from invitro situations, since there is presence of oral fluids and other consumed food. The decrease in effectiveness of the medications may be a result of loss of the material into the gel rather than resistance development in the organisms. ${ }^{6}$

In a review of treatment of oral candidosis, it was suggested that the antifungal agents, even at sub therapeutic concentrations, may be effective against virulence of yeast, especially intra orally where topical medication levels vary. This phenomenon was called post antifungal effect. ${ }^{10}$

The limitation of our study is that although we found effective activity of the agents, possible disadvantage is nonuniform particle size of the medication as a result of crushing the antifungal agents. Also, combining of the powdered 
medication with the tissue conditioner may result in differing concentrations of the medication throughout the mixture.

The optimum dosage of the drug has to be determined with further studies. The concentration of 5 micrograms used in the present study has proved to be effective; however, whether it is the optimum dose was not determined.

This study can be of clinical significance, as incorporation of antifungal drug ketoconazole and fluconazole into GC Soft and Viscogel shows good inhibition and can be recommended for clinical use. However, further clinical studies are required to quantify this and also to devise new modalities of drug dispensing in the form of liquids or ointments which could benefit the patients in the ease of application and drug delivery to the inflamed sites. These new methods of drug dispensing should enable adequate proportioning and mixing of the combinations in order to uniformly distribute the drug in the combinations, thus enabling a controlled and sustained effect of the medicaments in inhibiting Candida growth.

\section{CONCLUSIONS}

Tissue conditioners used in this study i.e. Viscogel and GC Soft do not have any antifungal property of their own. Both ketoconazole and fluconazole combinations with the tissue conditioners Viscogel and GC Soft are effective against Candida albicans. They can be used in the clinical treatment of established Candidal infection of mouth.

Data sharing statement provided by the authors is available with the full text of this article at jemds.com.

Financial or other competing interests: None.

Disclosure forms provided by the authors are available with the full text of this article at jemds.com.

\section{REFERENCES}

[1] Cumming CG, Wight C, Blackwell CL, et al. Denture stomatitis in the elderly. Oral Microbiol Immunol 1990;5(2):82-5.

[2] Webb BC, Thomas CJ, Whittle T. A 2-year study of Candidaassociated denture stomatitis treatment in aged care subjects. Gerodontology 2005;22(3):168-76.

[3] Urban VM, de Souza RF, Arrais CAG, et al. Effect of the association of nystatin with a tissue conditioner on its ultimate tensile strength. J Prosthodont 2006;15(5):2959.

[4] Chow KW, Matear W, Lawrence HP. Efficacy of antifungal agents in tissue conditioners in treating candidiasis. Gerodontology 1999;16(2):110-8.

[5] Yoshida K, Hirai K, Ara T, et al. Feasibility of using collagen as the base of the antifungal drug, miconazole. J Oral Rehabil 2006;33(3):363-7.

[6] Thomas CJ, Nutt GM. The in vitro fungicidal properties of Visco-gel, alone and combined with nystatin and amphotericin B. J Oral Rehab 1978;5(2):162-72.

[7] Iqbal Z, Zafar MS. Role of antifungal medicaments added to tissue conditioners: a systematic review. J Prosthodont Res 2016;60(4):231-9.

[8] Dorocka-Bobkowska B, Konopka K. Susceptibility of candida isolates from denture-related stomatitis to antifungal agents in vitro. Int $\mathrm{J}$ Prosthodont 2007;20(5):504-6.

[9] Quinn DM. The effectiveness, in vitro, of miconazole and ketoconazole combined with tissue conditioners in inhibiting the growth of Candida albicans. J Oral Rehabil 1985;12(2):172-82.

[10] Garcia-Cuesta C, Sarrion-Pérez MG, Bagán JV. Current treatment of oral candidiasis: a literature review. J Clin Exp Dent 2014;6(5):e576-82. 